\title{
THE HYPERSPACE OF A PSEUDOARC IS A CANTOR MANIFOLD ${ }^{1}$
}

\author{
TOOG NISHIURA AND CHOON-JAI RHEE
}

ABSTRACT. The following theorem which was conjectured by C. Eberhart and S. B. Nadler, Jr., in [EN] is proved.

THEOREM. The hyperspace of nonvoid subcontinua of a pseudoarc is a two-dimensional Cantor manifold.

1. Introduction. The hyperspace $C(X)$ of nonvoid subcontinua of a metric continuum $X$ has been investigated extensively. (We will restrict our discussion to metric continua.) It is known that $C(X)$ is always compact and arcwise connected [KE]. The basic work [S] establishes the relationship between $C(X)$ and inverse limit spaces. Inverse limit methods have yielded further properties of $C(X)$. Namely, $C(X)$ is acyclic in all dimensions $[\mathbf{S}]$, unicoherent $[\mathbf{S}],[\mathbf{N}]$, and has dimension exceeding one for nondegenerate $X[\mathbf{E N}]$. By specializing $X$, much more can be said of $C(X)$. Notable works along this line are [D1] and [D2] where $X$ is locally connected. The hyperspace of an hereditarily indecomposable continuum $X$ also has been studied. See [KE], [EN], [R], [T] and [H]. The present paper concerns itself with one such hereditarily indecomposable continuum, the pseudoarc. It is known that the hyperspace of a pseudoarc is embeddable in Euclidean three-dimensional space [T], $[\mathbf{H}]$ and that its dimension is two [EN]. We add to the large collection of facts about $C(X)$ the theorem stated in the abstract. This theorem improves the dimension two assertion of [EN].

2. The function $\mu$. Let $X$ be a nondegenerate metric continuum and $C(X)$ be the space of all nonvoid subcontinua of $X$ with the Hausdorff metric [KU]. In $[\mathbf{K E}]$, Kelley noted the existence (originally due to Whitney [W]) of a real-valued function $\mu$ defined on $C(X)$ and having

Presented to the Society, December 21, 1970; received by the editors February 10, 1971.

AMS 1970 subject classifications. Primary 54B20, 54F45. fold.

Key words and phrases. Pseudoarc, hyperspace of continua, dimension, Cantor mani-

${ }^{1}$ The first author was partially supported by the National Science Foundation Grant NSF GP-12915.

(c) American Mathematical Society 1972 
the following properties:

(1) $\mu$ is continuous;

(2) if $A, B \in C(X), A \subset B$ and $A \neq B$, then $\mu(A)<\mu(B)$;

(3) $\mu(X)=1$;

(4) $\mu(\{x\})=0$ for each $x \in X$.

He proved among others that

(a) $C(X)$ is an arcwise connected continuum;

(b) if $X$ is hereditarily indecomposable, $A, B \in C(X), A \cap B \neq \varnothing$ and $\mu(A)=\mu(B)$ then $A=B$;

(c) $X$ is hereditarily indecomposable if and only if $C(X)$ contains a unique arc between every pair of its elements.

Suppose further that $X$ is a pseudoarc. Then, in [R], it was observed that the space $\mu^{-1}(t), 0 \leqq t<1$, is a totally pathwise disconnected continuum. Subsequently, Eberhart and Nadler in [EN] observed that $\mu^{-1}(t)$ is a continuous decomposition of $X$ and hence, by [B1],

(d) $\mu^{-1}(t)$ is a pseudoarc for $0 \leqq t<1$ whenever $X$ is a pseudoarc.

We now prove three lemmas which will be needed later.

Lemma 2.1. Suppose $X$ is a pseudoarc and $0 \leqq t<1$. Then there is $a$ homeomorphism $h_{t}$ of $C(X)$ onto $\mu^{-1}[t, 1]=\{A \in C(X): t \leqq \mu(A)\}$ such that $h_{t}\left[\mu^{-1}(0)\right]=\mu^{-1}(t)$.

Proof. Using (d) above, we let $h: X \rightarrow \mu^{-1}(t)$ be a homeomorphism. Define a mapping $h$ on $C(X)$ onto the hyperspace $C\left(\mu^{-1}(t)\right)$ of the space $\mu^{-1}(t)$ by $\bar{h}(A)=h(A)$ for each $A \in C(X)$. Then $\bar{h}$ is a homeomorphism.

Let $2^{X}$ be the space of all nonvoid closed subsets of $X$ with the Hausdorff metric, and $2^{2^{X}}$ be the space of all nonvoid closed subsets of $2^{X}$ with Hausdorff metric. Let $\sigma: 2^{2^{X}} \rightarrow 2^{X}$ be defined by $\sigma(\mathscr{A})=\bigcup\left\{A \in 2^{X}: A \in \mathscr{A}\right\}$, $\mathscr{A} \in 2^{2^{X}}$. In [KE], it is shown that $\sigma$ is continuous. Since $C\left(\mu^{-1}(t)\right) \subset 2^{2^{x}}$, we let $\sigma$ be the restriction on $C\left(\mu^{-1}(t)\right)$. Let $\mathscr{A} \in C\left(\mu^{-1}(t)\right)$. Then $\sigma(\mathscr{A})$ is a subcontinuum of $X$, and thus $\sigma(\mathscr{A}) \in C(X)$. Let $A \in \mathscr{A}$. Then $t=\mu(A)$ and $A \subset \sigma(\mathscr{A})$, so that by the property (2) of $\mu, \mu(A) \leqq \mu(\sigma(\mathscr{A}))$. This implies that $\sigma(\mathscr{A}) \in \mu^{-1}[t, 1]$. If $A \in \mu^{-1}[t, 1]$, then $\mu(A)=s, s \geqq t$. Let $\mathscr{A}=$ $\left\{B \in \mu^{-1}(t): B \subset A\right\}$. For each $x \in A$, since the unique arc $\mathscr{A}_{x}$ in $C(X)$ from $\{x\}$ to $X$ must meet $\mu^{-1}(t)$, there is an element $B \in \mu^{-1}(t)$ such that $x \in B$ and $B \subset A$ [KE]. We would like to show that $\mathscr{A} \in C\left(\mu^{-1}(t)\right)$. Consider the projection mapping $f$ of $X$ onto the space $\mu^{-1}(t)$ defined by $f(x)=B$ if $x \in B$. This function is continuous [R], and $f(A)=\mathscr{A}$. Since $A$ is a subcontinuum of $X$, so is $\mathscr{A}$ in $\mu^{-1}(t)$. Therefore $\mathscr{A} \in C\left(\mu^{-1}(t)\right)$. Thus $\sigma(\mathscr{A})=A$ and $\sigma$ is a continuous mapping of $C\left(\mu^{-1}(t)\right)$ onto $\mu^{-1}[t, 1]$. The fact that $\sigma$ is oneto-one follows from (b) above. Therefore $\sigma: C\left(\mu^{-1}(t)\right) \rightarrow \mu^{-1}[t, 1]$ is a homeomorphism. 
We let $h_{t}: C(X) \rightarrow \mu^{-1}[t, 1]$ be the homeomorphism defined by $h_{t}=$ $\sigma \circ \bar{h}$. The lemma is now proved.

Lemma 2.2. Suppose $X$ is a pseudoarc and $0 \leqq t<1$. Then there is a mapping $g_{t}: C(X) \rightarrow C(X)$ such that $g_{t}$ restricted to $\mu^{-1}[t, 1]$ is $h_{t}^{-1}$ and $g_{t}\left[\mu^{-1}[0, t]\right]=\mu^{-1}(0)$.

Proof. For each $A \in \mu^{-1}[0, t]$ there is a unique set $B_{A} \in \mu^{-1}(t)$ such that $A \subset B_{A}$. Let $g_{t}(A)=h_{t}^{-1}\left(B_{A}\right)$. It is clear that $g_{t}$ is continuous on $\mu^{-1}[0, t]$. If $g_{t}$ is defined to be $h_{t}^{-1}$ on $\mu^{-1}[t, 1]$ then the desired mapping is constructed.

Since $\mu$ is a closed continuous mapping, we have immediately the following lemma.

Lemma 2.3. For each closed set $F$ and open set $\mathcal{O} \supset \mu^{-1}[F]$, there is an open set $Q$ such that $\mu^{-1}[F] \subset \mu^{-1}[Q] \subset \mathcal{O}$.

Finally, we remark that, if $h: C(X) \rightarrow C(X)$ is a homeomorphism and $X$ is a pseudoarc then necessarily $h\left[\mu^{-1}(0)\right]=\mu^{-1}(0)$ and $h(X)=X$.

3. The dimension of the hyperspace of a pseudoarc. In this section we prove two theorems concerning the dimension of the hyperspace $C(X)$ of a pseudoarc $X$. The first theorem has been established by Eberhart and Nadler [EN]. The present proof is new and relies only on properties of the pseudoarc. In the above-mentioned paper, it is observed that $C(X)$ is of dimension two at each point of $\mu^{-1}(0,1)=\{A \in C(X): 0<\mu(A)<1\}$. The second theorem of this section shows that $C(X)$ is also of dimension two at $X$. This fact will be used in the next section to prove the main theorem.

THEOREM 3.1. If $X$ is a pseudoarc then the dimension of $C(X)$ is two.

Proof. Since $C(X)$ is a nondegenerate continuum, we have $\operatorname{dim} C(X) \geqq$ 1. From Theorem VI.7 of [HW], we have

$$
\operatorname{dim} C(X) \leqq \operatorname{dim} \mu[C(X)]+\sup \left\{\operatorname{dim} \mu^{-1}(t): 0 \leqq t \leqq 1\right\} .
$$

From $\S 2$ above, we have that the right side of the above inequality is two since the dimension of a pseudoarc is one. We need to prove $\operatorname{dim} C(X) \neq 1$.

Suppose $\operatorname{dim} C(X)=1$. Since $C(X)$ is contractible [R], any mapping on a subcontinuum of $C(X)$ into $S^{\mathbf{1}}$ is inessential, and therefore each subcontinuum of $C(X)$ has property (b). Thus, each subcontinuum of $C(X)$ is unicoherent [WH, p. 226]. But there are subcontinua of $C(X)$ which are not unicoherent. For example, $\mu^{-1}(0) \cup \mathscr{A}_{x, y}$, where $\mathscr{A}_{x, y}$ is the unique arc in $C(X)$ between $\{x\}$ and $\{y\}, x \neq y, x, y \in X$. Since a pseudoarc contains no arc, $\mu^{-1}(0) \cap \mathscr{A}_{x, y}=\{x, y\}$. Consequently, $\operatorname{dim} C(X) \neq 1$ and the theorem is proved. 
THEOREM 3.2. If $X$ is a pseudoarc then $C(X)$ has dimension two at the point $X$.

Proof. The proof is by contradiction. Since $C(X)$ is a nondegenerate continuum, we have that the dimension of $C(X)$ at $X$ is no less than one. We will show that the assumption that $C(X)$ is of dimension one at the point $X$ implies $\operatorname{dim} C(X)=1$. The proof will be made in three parts.

Part 1. Let $0<t<1$. If $C(X)$ has dimension one at $X$ then there are two disjoint open sets $\mathscr{S}$ and $\mathscr{T}$ of $C(X)$ such that $\mu^{-1}(0) \subset \mathscr{S}$, $\mu^{-1}[t, 1] \subset \mathscr{T}$, and their boundaries have $\operatorname{dim} \operatorname{Bd}(\mathscr{S})=0=\operatorname{dim} \operatorname{Bd}(\mathscr{T})$.

Proof. Let $\mathcal{O}$ be an open neighborhood of $X$ such that $\mathcal{O} \subset \mu^{-1}\left[\frac{1}{2}, 1\right]$ and $\operatorname{dim} \operatorname{Bd}(\mathcal{O})=0$. Then, if $\mathscr{P}$ is the complement of the closure of $\mathcal{O}, \mathscr{P}$ is an open set containing $\mu^{-1}(0)$ and $\operatorname{dim} \operatorname{Bd}(\mathscr{P})=0$.

Let $P_{0} \in \mathcal{O}$ and $P_{0} \neq X$. As Eberhart and Nadler in [EN] observed, Theorem 15 of [B1] implies for each $A \in \mu^{-1}[t, 1], A \neq X$, there exists a homeomorphism $h_{A}: C(X) \rightarrow C(X)$ such that $h_{A}\left(P_{0}\right)=A$. Associated with this homeomorphism are two disjoint open sets $\mathcal{O}_{A}=h_{A}(\mathcal{O})$ and $\mathscr{P}_{A}=h_{A}(\mathscr{P})$ for which $A \in \mathcal{O}_{A}, \mu^{-1}(0) \subset \mathscr{P}_{A}$, and $\operatorname{dim} \operatorname{Bd}\left(\mathcal{O}_{A}\right)=0=\operatorname{dim} \operatorname{Bd}\left(\mathscr{P}_{A}\right)$. Now, $\left\{\mathcal{O}_{A}: A \in \mu^{-1}[t, 1], A \neq X\right\}$ is an open cover of the compact set $\mu^{-1}[t, 1]$. Let $\mathcal{O}_{A_{1}}, \cdots, \mathcal{O}_{A_{n}}$ be a subcover, $\mathscr{T}=\bigcup_{i=1}^{n} \mathcal{O}_{A_{i}}$ and $\mathscr{S}=\bigcap_{i=1}^{n} \mathscr{P}_{A_{i}}$. Then $\mathscr{S}$ and $\mathscr{T}$ are disjoint open sets with $\mu^{-1}(0) \subset \mathscr{S}$ and $\mu^{-1}[t, 1] \subset \mathscr{T}$. Since $\operatorname{Bd}(\mathscr{S}) \subset \bigcup_{i=1}^{n} \operatorname{Bd}\left(\mathscr{P}_{A_{i}}\right)$ and $\operatorname{Bd}(\mathscr{T}) \subset \bigcup_{i=1}^{n} \operatorname{Bd}\left(\mathcal{O}_{A_{i}}\right)$, we have $\operatorname{dim} \operatorname{Bd}(\mathscr{S})=0=\operatorname{dim} \operatorname{Bd}(\mathscr{T})$ and the first part is proved.

Part 2. Let $0 \leqq t \leqq 1$ and $\mathcal{O}$ be an open neighborhood of $\mu^{-1}(t)$. Suppose the conclusion of Part 1 holds. Then there is an open neighborhood $\mathscr{W}$ of $\mu^{-1}(t)$ such that $\mathscr{W} \subset \mathcal{O}$ and $\operatorname{dim} \operatorname{Bd}(\mathscr{W})=0$.

Proof. By Lemma 2.3, there are two numbers $s_{1}$ and $s_{2}$ such that $s_{1}<t<s_{2}$ and $\mu^{-1}\left[s_{1}, s_{2}\right] \subset \mathcal{O}$. We assume for convenience that $0<t<1$. The contrary cases involve only a slight modification of the argument. We may now further assume $0 \leqq s_{1}<t<s_{2} \leqq 1$.

Let us consider $s_{1}$. By Lemma 2.2 there is a mapping $g_{s_{1}}: C(X) \rightarrow C(X)$ such that $g_{s_{1}}$ maps $\mu^{-1}\left[s_{1}, 1\right]$ homeomorphically onto $C(X)$ and $g_{s_{1}}\left[\mu^{-1}\left[0, s_{1}\right]\right]=\mu^{-1}(0) . g_{s_{1}}\left[\mu^{-1}[t, 1]\right]$ is a closed set disjoint with $\mu^{-1}(0)$. Hence by Lemma 2.3 there is a number $T_{1}$ with $0<T_{1}$ such that $\mu^{-1}\left[0, T_{1}\right] \cap g_{s_{1}}\left[\mu^{-1}[t, 1]\right]=\varnothing$. From Part 1 there is an open set $\mathscr{T}$ such that the closure of $\mathscr{T}$ does not meet $\mu^{-1}(0), \mathscr{T} \supset \mu^{-1}\left[T_{1}, 1\right]$ and $\operatorname{dim} \operatorname{Bd}(\mathscr{T})=0$. Thus, if $\mathscr{W}_{1}=g_{s_{1}}^{-1}(\mathscr{T})$ then $\mathscr{W}_{1}$ is open, $\mu^{-1}(t) \subset \mathscr{W}_{1} \subset$ $\mu^{-1}\left[s_{1}, 1\right]$ and $\operatorname{dim} \operatorname{Bd}\left(\mathscr{W}_{1}\right)=0$.

Next, consider $t$. By Lemma 2.2 there is a mapping $g_{t}: C(X) \rightarrow C(X)$ such that $g_{t}$ maps $\mu^{-1}[t, 1]$ homeomorphically onto $C(X)$ and $g_{t}\left[\mu^{-1}[0, t]\right]=\mu^{-1}(0) . g_{t}\left[\mu^{-1}\left[s_{2}, 1\right]\right]$ is a closed set disjoint with $\mu^{-1}(0)$. Hence by Lemma 2.3 there is a number $T_{2}$ with $0<T_{2}$ such that 
$\mu^{-1}\left[0, T_{2}\right] \cap g_{t}\left[\mu^{-1}\left[s_{2}, 1\right]\right]=\varnothing$. From Part 1 , there is an open set $\mathscr{S}$ such that the closure of $\mathscr{S}$ does not meet $\mu^{-1}\left[T_{2}, 1\right], \mathscr{S} \supset \mu^{-1}(0)$ and $\operatorname{dim} \operatorname{Bd}(\mathscr{S})=0$. Thus, if $\mathscr{W}_{2}=g_{t}^{-1}(\mathscr{S})$ then $\mathscr{W}_{2}$ is open, $\mu^{-1}(t) \subset \mathscr{W}_{2} \subset$ $\mu^{-1}\left[0, s_{2}\right]$ and $\operatorname{dim} \operatorname{Bd}\left(\mathscr{W}_{2}\right)=0$.

Let $\mathscr{W}=\mathscr{W}_{1} \cap \mathscr{W}_{2}$. Then $\mathscr{W}$ is open, $\mu^{-1}(t) \subset \mathscr{W} \subset \mu^{-1}\left[s_{1}, s_{2}\right] \subset \mathcal{O}$ and $\operatorname{dim} \operatorname{Bd}(\mathscr{W}) \leqq \operatorname{dim} \operatorname{Bd}\left(\mathscr{W}_{1}\right)+\operatorname{dim} \operatorname{Bd}\left(\mathscr{W}_{2}\right)=0$. Thus Part 2 is proved.

Part 3. If $C(X)$ has dimension one at $X$ then $\operatorname{dim} C(X)=1$.

Proof. Let $\mathscr{K}=\left\{\mu^{-1}(t): 0 \leqq t \leqq 1\right\}$. Then $\mathscr{K}$ is a family of closed subsets of $C(X)$. By Part 2, each neighborhood of $\mu^{-1}(t)$ contains a neighborhood whose boundary has dimension zero. Since $\operatorname{dim} \mu^{-1}(t) \leqq 1$ for each $t$, we have, by Proposition $\mathrm{G}$ on p. 90 of $[\mathrm{HW}], \operatorname{dim} C(X)=$ $\operatorname{dim} \bigcup \mathscr{K} \leqq 1$, a contradiction to Theorem 3.1. Thus Theorem 3.2 is proved.

4. Proof of the main theorem. We are now in a position to prove our main theorem. Lemma 2.3 provides us with the fact that the family $\mu^{-1}[t, 1], 0 \leqq t<1$, forms a basis of closed neighborhoods of the point $X$ in $C(X)$. We infer from Lemma 2.1 that we need only consider the neighborhood $C(X)$.

THEOREM 4.1. If $X$ is a pseudoarc then $C(X)$ is a two-dimensional Cantor manifold.

Proof. By denying the conclusion, we will establish a contradiction to Theorem 3.2. That is, we will show that the existence of a zero-dimensional separator of $C(X)$ implies the existence of an open neighborhood of $X$, disjoint with $\mu^{-1}(0)$, whose boundary has dimension zero. Then the preliminary remarks of this section will complete the proof.

Suppose $\mathscr{S}$ is a closed zero-dimensional subset of $C(X)$ which separates $C(X)$. Let $\mathscr{A}$ and $\mathscr{B}$ be nonvoid open sets such that $C(X)-\mathscr{S}=\mathscr{A} \cup \mathscr{B}$. We will consider two cases.

Case 1. Suppose $X \notin \mathscr{S}$. Without loss of generality, we may assume $X \in \mathscr{A}$. There are now two possibilities. Either $\mathscr{S} \cap \mu^{-1}(0)=\varnothing$ or $\mathscr{S} \cap \mu^{-1}(0) \neq \varnothing$. Let us dispose of the first possibility.

(a) Suppose $\mathscr{S} \cap \mu^{-1}(0)=\varnothing$. In the event that $\mu^{-1}(0) \subset \mathscr{B}$, the desired neighborhood of $X$ is $\mathscr{A}$ and the contradiction is established. Since $\mu^{-1}(0)$ is connected, $\mu^{-1}(0) \nsubseteq \mathscr{B}$ implies $\mu^{-1}(0) \subset \mathscr{A}$. $\mathscr{B}$ being nonvoid, choose $P \in \mathscr{B} . P$ is a nondegenerate subcontinuum of $X$ since $P \notin \mu^{-1}(0)$. Hence $P$ is a pseudoarc. $C(P)$ is homeomorphic to $C(X)$ and $C(P)$ is a subspace of $C(X)$. Clearly, $\mathscr{B} \cap C(P)$ is an open neighborhood of $P$ in $C(P)$, disjoint with $C(P) \cap \mu^{-1}(0)=\{\{p\}: p \in P\}$, whose boundary in $C(P)$ has dimension zero. Hence, the required neighborhood of $X$ exists and the 
contradiction is established. Thus, we have disposed of the possibility $\mathscr{S} \cap \mu^{-1}(0)=\varnothing$.

(b) Suppose $\mathscr{S} \cap \mu^{-1}(0) \neq \varnothing$. Either $\mathscr{B} \cap \mu^{-1}(0) \neq \varnothing$ or $\mathscr{B} \cap \mu^{-1}(0)=\varnothing$. Suppose first that $\mathscr{B} \cap \mu^{-1}(0) \neq \varnothing$. Let $P_{0} \in \mathscr{B} \cap \mu^{-1}(0)$ and $A \in \mu^{-1}(0)$. Then, both $P_{0}$ and $A$ are singleton subsets of $X$. Since $X$ is homogeneous, there is a homeomorphism $h_{A}: C(X) \rightarrow C(X)$ such that $h_{A}\left(P_{0}\right)=A$. Associated with each such homeomorphism are two disjoint open sets $\mathcal{O}_{A}=h_{A}(\mathscr{A})$ and $\mathscr{P}_{A}=h_{A}(\mathscr{B})$ with the properties $X \in \mathcal{O}_{A}$ and $\operatorname{dim} \operatorname{Bd}\left(\mathscr{O}_{A}\right)=0$. Since $\left\{\mathscr{P}_{A}: A \in \mu^{-1}(0)\right\}$ is an open cover of the compact set $\mu^{-1}(0)$, there is a finite cover $\mathscr{P}_{A_{1}}, \cdots, \mathscr{P}_{A_{n}}$. Let $\mathcal{O}=\bigcap_{i=1}^{n} \mathcal{O}_{A_{i}}$ and $\mathscr{P}=\bigcup_{i=1}^{n} \mathscr{P}_{A_{i}}$. Then $\mathcal{O}$ and $\mathscr{P}$ are disjoint open sets, $X \in \mathcal{O}, \mu^{-1}(0) \subset \mathscr{P}$ and $\operatorname{dim} \operatorname{Bd}(\mathcal{O})=0$. Thus, the desired neighborhood of $X$ is found and the contradiction established. Next, suppose $\mathscr{B} \cap \mu^{-1}(0)=\varnothing$. Since $X \notin \mathscr{B}$ and $\mathscr{B} \neq \varnothing$, there is a nondegenerate subcontinuum $P \in \mathscr{B}$. $P$ is a pseudoarc and $C(P)$ is a subspace of $C(X)$ which is homeomorphic to $C(X)$. Since $\operatorname{dim}\left[C(P) \cap \mu^{-1}(0)\right]=1$ and $\operatorname{dim} \mathscr{S}=0$, we have $\left[C(P) \cap \mu^{-1}(0)\right]-\mathscr{S}$ is a nonempty subset of $\mathscr{A} \cap C(P)$. By considering $C(P), \mathscr{S}^{\prime}=C\left({ }^{D}\right) \cap \mathscr{S}, \mathscr{A}^{\prime}=\mathscr{B} \cap C(P)$ and $\mathscr{B}^{\prime}=\mathscr{A} \cap C(P)$, we see that $\mathscr{S}^{\prime}$ is a zero-dimensional separator of $C(P), C(P)-\mathscr{S}^{\prime}=$ $\mathscr{A}^{\prime} \cup \mathscr{B}^{\prime}$ where $\mathscr{A}^{\prime}$ and $\mathscr{B}^{\prime}$ are open sets, $P \in \mathscr{A}^{\prime}$ and $\mathscr{B}^{\prime} \cap \mu_{P}^{-1}(0) \neq \varnothing$ where $\mu_{P}$ is a $\mu$ function associated with the pseudoarc $P$. We have arrived at the situation which immediately preceded the one at hand.

Now the two possibilities (a) and (b) under Case 1 have been completely disposed of.

Case 2. Suppose $X \in \mathscr{S}$. We will dispose of this case by reducing it to Case 1.

For each $x \in X$, there is a unique arc $\mathscr{A}_{x}$ in $C(X)$ from $\{x\}$ to $X$ [KE]. Let $M=\left\{x \in X: \mathscr{A}_{x} \cap \mathscr{A} \neq \varnothing\right\}$ and $N=\left\{x \in X: \mathscr{A}_{x} \cap \mathscr{B} \neq \varnothing\right\}$. Since $\operatorname{dim} \mathscr{S}=$ 0 , we have $\varnothing \neq \mathscr{A}_{x}-\mathscr{S} \subset \mathscr{A} \cup \mathscr{B}$ for each $x \in X$. Consequently, $X=M \cup N$. We will show $M \neq \varnothing$ and open. A symmetric argument shows $N \neq \varnothing$ and open. To this end, we recall a continuous mapping $\Phi: X \times[0,1] \rightarrow$ $C(X)$ defined in Theorem 3.5 of $[\mathbf{R}] . \Phi$ is defined as

$$
\Phi(x, t)=A, \text { where } x \in A \in C(X) \text { and } \mu(A)=t .
$$

Since each pair of points in $C(X)$ has a unique arc between them, we have $\mathscr{A}_{x}=\Phi[\{x\} \times[0,1]]$. Consequently, $M=F\left[\Phi^{-1}(\mathscr{A})\right]$, where $F$ is the natural projection $F: X \times[0,1] \rightarrow X$.

Since $X$ is connected $M \cap N \neq \varnothing$. Let $x \in M \cap N$ and $P \in \mathscr{A}_{x} \cap \mathscr{A}$ and $Q \in \mathscr{A}_{x} \cap \mathscr{B}$. Since $P$ and $Q$ are in the arc $\mathscr{A}_{x}$, either $P \supset Q$ or $P \subset Q$. Also, $P \neq Q$. Suppose $P \supset Q$. By considering the pseudoarc $P$, we have for $C(P)$, $\mathscr{S}^{\prime}=C(P) \cap \mathscr{S}, \mathscr{A}^{\prime}=\mathscr{A} \cap C(P), \mathscr{B}^{\prime}=\mathscr{B} \cap C(P)$, precisely the Case 1. Similar considerations apply when $P \subset Q$.

The main theorem is now established. 


\section{BIBLIOGRAPHY}

[B1] R. H. Bing, A homogeneous indecomposable plane continuum, Duke Math. J. 15 (1948), 729-742. MR 10, 261.

[B2] — Concerning hereditarily indecomposable continua, Pacific J. Math. 1 (1951), 43-51. MR 13, 265.

[D1] R. Duda, On the hyperspace of subcontinua of a finite graph. I, Fund. Math. 62 (1968), 265-286. MR 38 \#5175a.

[D2] — On the hyperspace of subcontinua of a finite graph. II, Fund. Math. 63 (1968), 225-255. MR 38 \#5175b.

[D3] - Correction to the paper "On the hyperspace of subcontinua of a finite graph. I," Fund. Math. 69 (1970), 207-211.

[EN] C. Eberhart and S. B. Nadler, Jr., The dimension of certain hyperspaces, Bull. Acad. Polon. Sci. (to appear).

[H] G. W. Henderson, On the hyperspace of subcontinua of arc-like continua, Proc. Amer. Math. Soc. 27 (1971), 416-417.

[HW] W. Hurewicz and H. Wallman, Dimension theory, Princeton Math. Series, vol. 4, Princeton Univ. Press, Princeton, N.J., 1941. MR 3, 312.

[KE] J. Kelley, Hyperspaces of a continuum, Trans. Amer. Math. Soc. 52 (1942), 22-36. MR 3, 315.

[KU] K. Kuratowski, Topology. Vol. II, Academic Press, New York, 1968. MR 41 \#4467.

[N] S. B. Nadler, Jr., Inverse limits and multicoherence, Bull. Amer. Math. Soc. 76 (1970), 411-414. MR 40 \#6514.

[R] C. J. Rhee, On dimension of hyperspace of a metric continuum, Bull. Soc. Roy. Sci. Liège 38 (1969), 602-604.

[S] J. Segal, Hyperspaces of the inverse limit space, Proc. Amer. Math. Soc. 10 (1959), 706-709. MR 21 \#7492.

[T] W. R. Transue, On the hyperspace of subcontinua of the pseudoarc, Proc. Amer. Math. Soc. 18 (1967), 1074-1075. MR 36 \#5901.

[WH] H. Whitney, Analytic topology, Amer. Math. Soc. Colloq. Publ., vol. 28, Amer. Math. Soc., Providence, R.I., 1942.

Department of Mathematics, Wayne State University, Detroit, Michigan 48202 